\title{
The activity of thalamic nucleus reuniens is critical for memory retrieval, but not essential for the early phase of "off-line" consolidation
}

\author{
Hao Mei, ${ }^{1}$ Nikos K. Logothetis, ${ }^{1,2}$ and Oxana Eschenko ${ }^{1}$ \\ ${ }^{7}$ Max Planck Institute for Biological Cybernetics, Tübingen 72076, Germany; ${ }^{2}$ Centre for Imaging Sciences, Biomedical Imaging \\ Institute, The University of Manchester, Manchester M13 9PT, United Kingdom
}

\begin{abstract}
Spatial navigation depends on the hippocampal function, but also requires bidirectional interactions between the hippocampus (HPC) and the prefrontal cortex (PFC). The cross-regional communication is typically regulated by critical nodes of a distributed brain network. The thalamic nucleus reuniens (RE) is reciprocally connected to both HPC and PFC and may coordinate the information flow within the HPC-PFC pathway. Here we examined if RE activity contributes to the spatial memory consolidation. Rats were trained to find reward following a complex trajectory on a crossword-like maze. Immediately after each of the five daily learning sessions the RE was reversibly inactivated by local injection of muscimol. The post-training RE inactivation affected neither the spatial task acquisition nor the memory retention, which was tested after a 20-d "forgetting" period. In contrast, the RE inactivation in well-trained rats prior to the maze exposure impaired the task performance without affecting locomotion or appetitive motivation. Our results support the role of the RE in memory retrieval and/or "online" processing of spatial information, but do not provide evidence for its engagement in "off-line" processing, at least within a time window immediately following learning experience.
\end{abstract}

[Supplemental material is available for this article.]

The interactions between the hippocampus (HPC) and the prefrontal cortex (PFC) are essential for many aspects of spatial cognition, particularly those engaging working or declarative-like memory (Buzsáki 1996; Benchenane et al. 2011; Gordon 2011; Eichenbaum 2017). It has been shown, for instance, that the oscillatory rhythms generated in the HPC may synchronize firing of the PFC neurons (Hyman et al. 2005; Siapas et al. 2005). The HPC-PFC oscillatory coupling accompanies rule-guided behaviors (Jones and Wilson 2005; Benchenane et al. 2010), but is also observed during "off-line" states (e.g., sleep), when fluctuations of the hippocampal and cortical population excitability (indicated by ripples and sleep spindles) are coordinated by cortical slow rhythms (Siapas and Wilson 1998; Moelle et al. 2009; Wierzynski et al. 2009). The HPC-PFC interlay occurring "off-line" is thought to underlie the information transfer from the HPC, where new information is first encoded, to the PFC and other neocortical regions for the longterm storage (Buzsáki 1996; Frankland and Bontempi 2005; Peyrache et al. 2009; Colgin 2011). The HPC-PFC interactions are supported by their anatomical connectivity, including reciprocal direct projections. In the rodent brain, the CA1 subfield of ventral HPC and the ventral subiculum (main HPC output) project to the prelimbic and infralimbic areas of the PFC (Thierry et al. 2000; Hoover and Vertes 2007). The top-down prefrontal (predominantly anterior cingulate) projections target the CA1 and CA3 subfields of the dorsal HPC; this recently identified direct PFC-HPC pathway has been implicated in the contextual fear memory retrieval (Rajasethupathy et al. 2015). Given the variety and complexity of cognitive processes that appear to depend on HPC-PFC interac-

Corresponding author: oxana.eschenko@tuebingen.mpg.de

Article is online at http://www.learnmem.org/cgi/doi/10.1101//m.047134. 117. tions and the widespread anatomical connectivity of each structure to other brain regions, it is evident that the neural circuits supporting the performance of cognitively demanding tasks include brain structures beyond these two regions (Mizumori et al. 2000). Identifying the critical "hubs" of large-scale functional networks would advance our understanding of the neurophysiological mechanisms related to cognitive capacities, such as memory encoding, consolidation, and its long-term storage.

The nucleus reuniens (RE) is one such potentially critical node. As part of the midline thalamus, RE has been hypothesized to play a role for the HPC-PFC interactions (Vertes 2006; Vertes et al. 2007; Varela et al. 2014). This hypothesis originated from the studies of anatomical connectivity between the RE, HPC, and PFC and by now has received some support from functional studies. The RE is reciprocally connected to all subregions of the PFC, it densely projects to the dorsal and ventral HPC and also receives afferents from the ventral HPC and subiculum (McKenna and Vertes 2004; Vertes et al. 2006; Hoover and Vertes 2007). Notably, some of the RE neurons send their collaterals to both brain regions (Hoover and Vertes 2012). Such an anatomical connectivity pattern evidently places the RE in a key position for regulating the information flow between HPC and PFC (Vertes et al. 2007; Griffin 2015; Hallock et al. 2016; Roy et al. 2017). Indeed, RE lesion and/or inactivation impairs performance of previously learnt spatial and nonspatial tasks, particularly those requiring working memory (Davoodi et al. 2009; Hembrook and Mair 2011; Hembrook et al.

(C) 2018 Mei et al. This article is distributed exclusively by Cold Spring Harbor Laboratory Press for the first 12 months after the full-issue publication date (see http://learnmem.cshlp.org/site/misc/terms.xhtml). After 12 months, it is available under a Creative Commons License (Attribution-NonCommercial 4.0 International), as described at http://creativecommons.org/licenses/by-nc/4.0/. 
2012; Hallock et al. 2013, 2016; Layfield et al. 2015). Nonetheless, the role of RE for spatial learning remains controversial as several studies were unable to demonstrate any effects of RE lesion or inactivation on the rat ability to acquire a spatial task (Dolleman-van der Weel et al. 2009; Loureiro et al. 2012; Ito et al. 2015), but see also (Davoodi et al. 2009). Furthermore, despite the lack of evidence that RE neurons encode spatial representations, the RE output appears to affect the hippocampal spatial coding (Ito et al. 2015). The "head direction" coding by a population of RE neurons may also contribute to spatial navigation (Jankowski et al. 2014). At present, little is known about the role of RE for the HPC-PFC interaction occurring "off-line." Experimental evidence exists that the RE may be involved in the spatial memory consolidation (Davoodi et al. 2009) and/or its long-term maintenance (Loureiro et al. 2012; Ali et al. 2017). The results from studies on aversive learning also suggest that the RE may be an intermediate processing step within the PFC-HPC pathway that enables fear memory consolidation and retrieval (Davoodi et al. 2011; Xu and Sudhof 2013). Yet, in contrast to the well-established role of the HPC-PFC interactions for both "online" and "off-line" memoryrelated processing, the exact role and the functional significance of RE activity within this highly interconnected neural circuit in different stages of memory formation remains insufficiently understood.

The present study was thus designed to examine the extent to which the RE is involved in the consolidation of spatial memory. To this end, we trained rats to perform a spatial task and evaluated the behavioral effects of post-learning reversible inactivation of the RE. From a methodological perspective, inactivation of a brain region after encoding phase (or "online" processing) tests its involvement in the consolidation phase, taking place "off-line." We also tested rat spatial memory after 20-d "forgetting" period; any deviation of the rat performance on the remote memory test would indicate the involvement of post-learning RE activity in memory retention.

Rats were trained on an elevated crossword-like maze (Fig. $1 \mathrm{~A})$, the configuration of which resembled a multiple-unit T-alley maze, originally designed to study rodent spatial cognition (Tolman 1948). In the beginning of each trial a rat was randomly released from one of the two start locations and allowed to reach reward by navigating along maze alleys (Fig. 1A). Two salient distal visual cues were fixed on the black curtains surrounding the maze. The experimental design, namely, availability of distal cues, variable start positions, and goal-oriented navigation along the two rather complex trajectories, assumed that the rat's performance will be relying on allocentric cues and a path integration, both known to depend on HPC (McNaughton et al. 2006; Buzsáki and Moser 2013). Performing this maze task relying on procedural memory (e.g., acquired motor habit) is unlikely at the early stages of learning, but it may eventually prevail following extensive training (Packard and McGaugh 1996), which however did not take place in the present study. Based on the literature reviewed above, efficient learning and successful performance of such a spatial task most likely require a coordinated interplay between HPC and PFC, whereas the "off-line" HPC-PFC interactions may be essential for the stabilization of encoded information.

Our present findings demonstrate that RE activity is essential for the performance of a spatial target-oriented spatial task, however not required for the early phase of "off-line" processing enabling memory stabilization and long-term storage. In other words, the contribution of the RE is likely limited to the network that is activated "online" or during active phases of information encoding.

\section{Results}

Rats were trained on the crossword-like maze (Fig. 1A). The rat performance on the maze was evaluated using the trial latency (time required to reach reward), the trajectory length (total number of maze alleys visited by a rat before reaching reward), and the number of errors. Each deviation from the "correct" (shortest) path was considered an error, regardless of how many maze alleys the rat would cross before returning to the "correct" path (see example on Supplemental Fig. S1A). Traversing along the "correct" path, but in the opposite direction was also classified as an error (Supplemental Fig. S1B). Entering a "wrong" maze alley with all four paws was considered an error.

Immediately after each of the five learning sessions, muscimol (MUS) or saline (SAL) was injected via chronically implanted cannulas targeting the RE (Fig. 1B). On days 6 and 7, MUS or SAL was injected prior to the maze exposure to test the effect of RE inactivation on the performance of a recently acquired spatial task (Fig. 1B). On day 8 , we tested the drug-free rats on the maze again, but included two probe trials to verify that rats used distal visual cues for navigation on the maze. Specifically, the first three trials were run under the standard conditions, then all lights were turned off and rats had to perform the trial 4 in the darkness. Next trials (5-7) were standard and on the trial 8 rats were tested in the
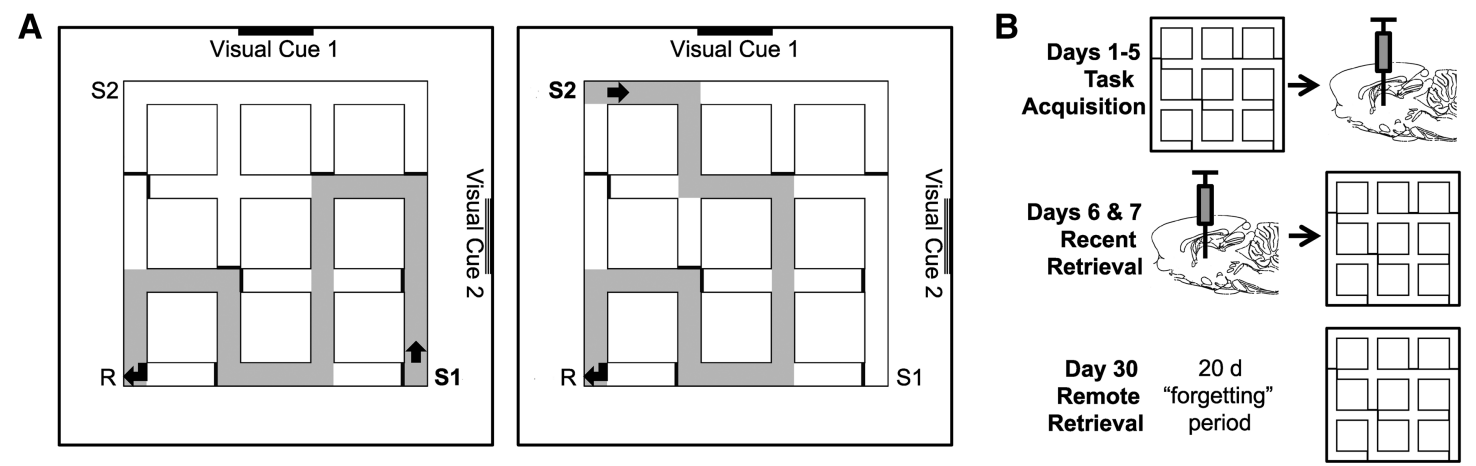

Figure 1. The experimental setup. (A) Top-down view of the experimental environment. The crossword-like maze consisted of perpendicular alleys; vertical barriers (thick black lines) blocked the access to some maze sections. There were two start locations (S1, S2) and one reward port (R). The "correct" (shortest) trajectory from Start 1 (left panel) and from Start 2 (right panel) are shown in gray; arrows indicate the direction of navigation. Two distal large visual cues were fixed on the black curtains (outer frame) surrounding the maze. (B) The experiment timeline. (Top) During the task acquisition sessions (days 1-5) rats received intrabrain injection of MUS or SAL immediately after the maze exposure. Middle, On days 6 and 7 the drug (MUS or SAL) was injected $30 \mathrm{~min}$ prior to the maze exposure. Bottom, After two additional injection-free training sessions on days 8 and 9 (not shown), rats were allowed $20 \mathrm{~d}$ of "forgetting" period and tested on the maze again on day 30. 
darkness and without any vertical barriers that prevented them entering some maze alleys during the initial learning (Fig. 1A); the trials 9 and 10 were again standard. On trial 4 (darkness test), rats made significantly more errors (Supplemental Fig. S2), which was suggestive that rats used allocentric, but not procedural (e.g., sequence of turns) strategy to perform the task. On trial 8 (no barriers test), all rats easily reached reward, but followed completely different trajectories from the "correct" ones (Supplemental Fig. S3); thus rats did not appear to rely on the acquired motor habit (e.g., sequence of turns), but likely used path integration. On day 9 , rats were trained in the standard conditions to stabilize the task performance and were tested on the maze again after 20-d "forgetting" period (Fig. 1B).

The histological examination confirmed the location of the cannula tip in the direct proximity to the RE in 17 rats (Fig. 2). The cases with the cannula placement outside the RE served as additional control. The histology result was in agreement with a post hoc analysis of the presence or absence of a motor deficit following intrabrain MUS injection. Typically, the RE inactivation did not affect motor activity. In contrast, MUS injection outside the RE or into the third ventricle often produced a motor deficit of different degrees. To quantify motor activity, for each of 10 trials after the MUS injections we extracted the rat maximal speed using video recording and a custom software (MathWorks). The K-means clustering analysis revealed three main patterns with motor activity stable across trials (Cluster1, Supplemental Fig. S4), gradually decreasing over time (Cluster2), and severely suppressed (Cluster3). In two rats with the cannula tip placed in the RE, the onset of motor deficit was delayed, probably due to MUS diffusion outside the RE. Conservatively, the data from these two rats were excluded from the analysis of the effects of the RE inactivation on memory consolidation. Hence, the rats were distributed between three experimental conditions with MUS injections restricted to the RE (RE-MUS, $n$ $=7$ ), with MUS injections outside of RE (notRE-MUS, $n=7$ ) and SAL injections (SAL, $n=16$ ). During the first training session (before any intrabrain injections), the rats' behavior on the maze was similar across three experimental groups (one-way analysis of variance [ANOVA]; errors: $\left(F_{(2,27)}=0.39\right.$, ns; trial latency: $F_{(2,27)}=1.32$, ns; trajectory length: $\left.F_{(2,27)}=0.24, \mathrm{~ns}\right)$.

\section{The RE is not essential for "off-line" memory consolidation or long-term storage}

To assess the effect of post-learning inactivation of the RE on the learning rate, we submitted behavioral variables across five training days to the repeated-measures ANOVA and compared across three experimental conditions. There was a significant day effect for the trial latency $\left(F_{(2.7,71.6)}=154.5, P<0.001\right.$; Greenhouse-Geisser correction was applied whenever the assumption of sphericity was violated), the trajectory length $\left(F_{(2.4,65.3)}=84.3, P<0.001\right)$ and the number of errors $\left(F_{(4,108)}=184.5, P<0.001\right)$, yet no significant interaction or group effect. The task performance gradually improved over time as reflected by decreasing number of errors, but the learning rate was equal for all three groups (Fig. 3A). Post hoc multiple comparisons (Bonferroni corrected) between training days showed that rats learned the task during four sessions; the number of errors reached an asymptote level on day 4 and there was no further improvement on day 5 (Fig. 3B). There was also no between-group difference on the last day of training (one-way ANOVA, errors: $F_{(2,27)}$ $=1.25$, ns; trial latency: $F_{(2,27)}=1.44$, ns; trajectory: $F_{(2,27)}=1.40$, ns). Since the rats had to learn two partially overlapping trajectories (Fig. 1A), we also compared the learning rate between Start1and Start2-trials. No trajectory preference was revealed in any experimental group by any of the behavioral variables analyzed (not shown). Finally, to evaluate the efficiency of spatial memory consolidation, we compared the rat performance during the first trial on days $2-5$, which is equivalent to the conventional memory retrieval test. We found a significant day effect (latency: $F_{(3,81)}=$ $34.14, P<0.001$; errors: $F_{(3,81)}=19.79, P<0.001$; trajectory: $F_{(3,81)}$ $=11.04, P<0.001)$, but no interaction or group effect. Overall, our results did not provide any evidence that post-learning inactivation of the RE affected the learning rate. Notably, post-learning MUS injections outside the RE, while caused a transient motor deficit, did not affect the next day task performance; the latter result suggests that brain regions surrounding the RE are not likely to contribute to the "off-line" consolidation.

We also tested if post-learning inactivation of the RE affected the long-term stability of acquired memory. After the initial learning phase was completed (days 1-5), rats were additionally tested
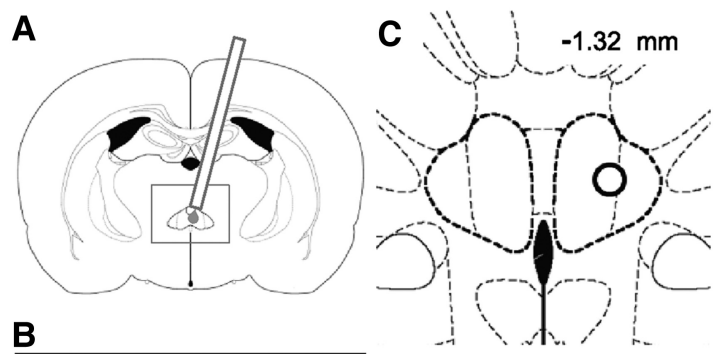

E

$\because-1.72 \mathrm{~mm} \quad \mathrm{G}$

G $\quad-1.92 \mathrm{~mm}$

B
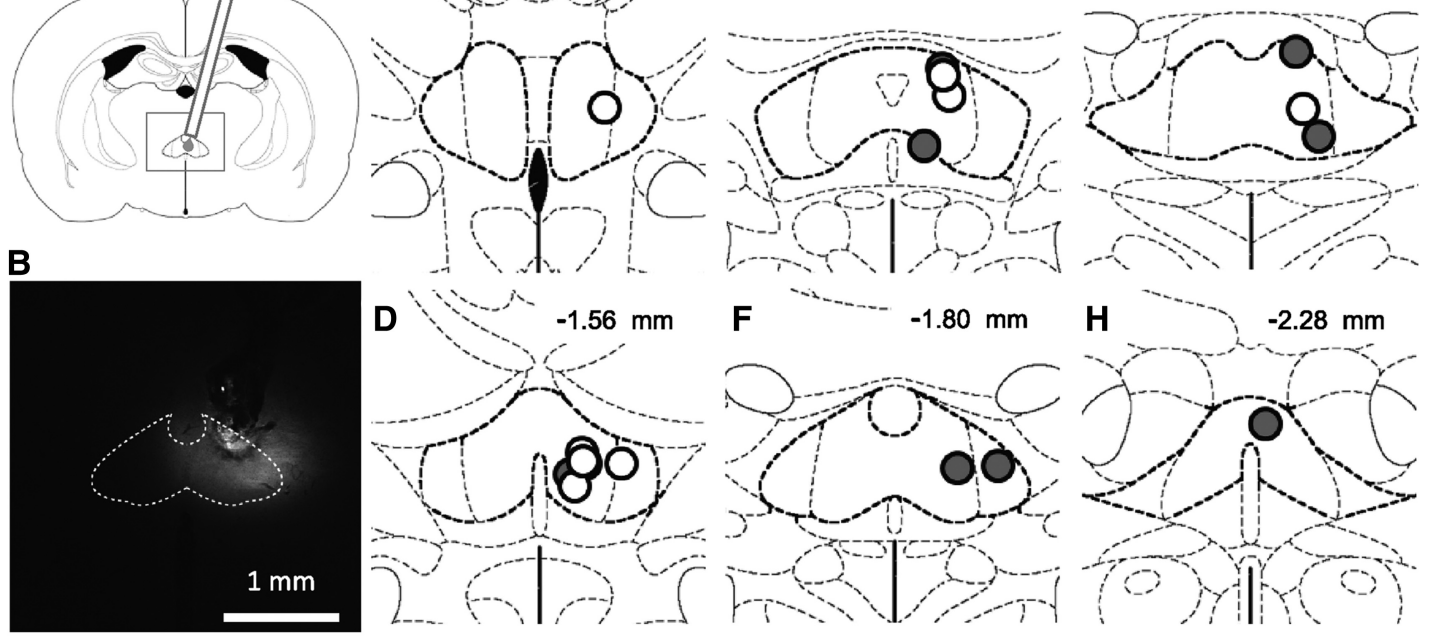

Figure 2. Histological reconstruction of the injection sites in the RE. $(A)$ Schematic of the rat brain coronal section with injection cannula targeting the RE. $(B)$ Enlarged brain section (indicated in $A$ ) showing the spread of fluorophore-conjugated MUS. Dashed line shows the RE boarders. ( $C-H$ ) Reconstruction of the injection centers in the RE on different anterior-posterior planes. Placements of the post-learning injections of SAL (open circles) and MUS (filled circles) within the RE are shown; cases with injection centers outside the RE are not shown. 

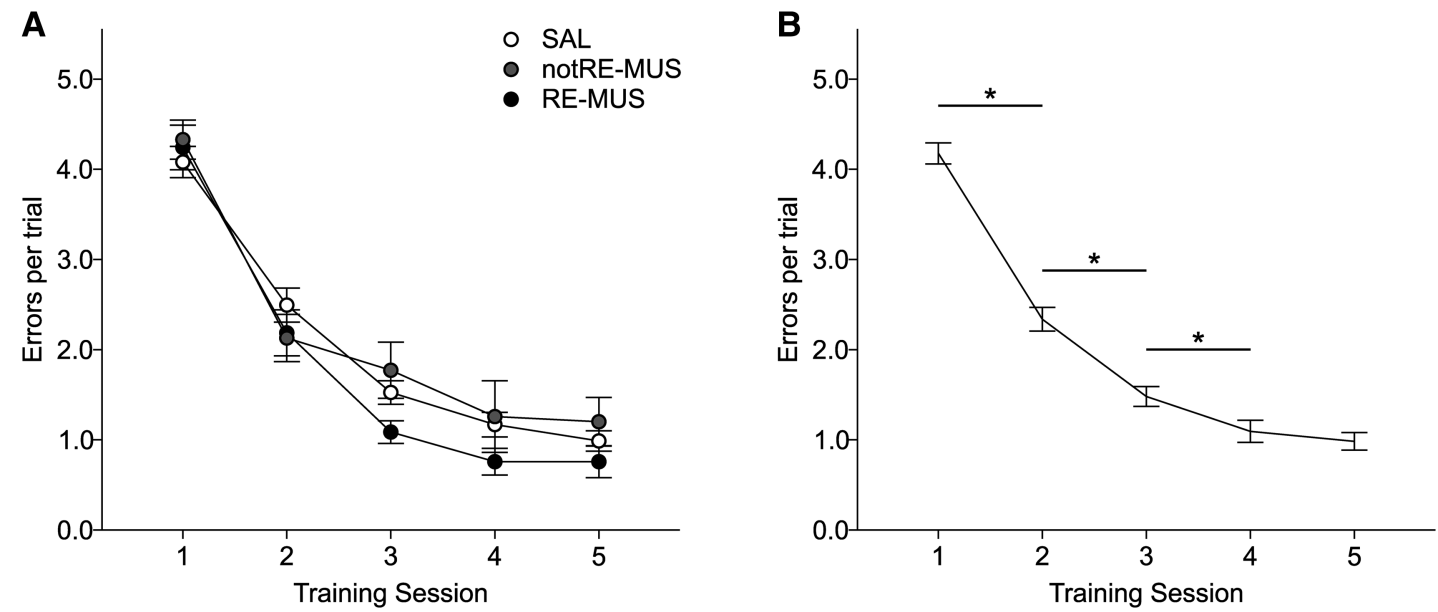

Figure 3. Reversible inactivation of the RE after each of the five task acquisition sessions did not affect the efficiency of spatial learning. ( $A$ ) The average number of errors in each trial is shown across five training sessions for rats that received intrabrain injections of SAL (open circles, $n=16$ ), MUS outside the $\mathrm{RE}$ (gray circles, $n=7$ ), or MUS in the RE (black circles, $n=7$ ). The accuracy of rat performance improved with equal rate in each experimental group. (B) The average number of errors in each trial is shown across five training sessions for all rats tested $(n=30)$. Rats reached asymptote performance on day 4 . Error bars represent \pm SEM; $\left({ }^{*}\right) P<0.01$ (Bonferroni corrected).

on the maze on days 6-9 and then were allowed $20 \mathrm{~d}$ of "forgetting" period, during which rats were kept in their home cages with unlimited access to food and water (Fig. 1B). At the end of the forgetting period rats were food deprived and tested again on the maze. The remote memory retrieval was evaluated by rat performance on the first trial. No intrabrain injections were made during "forgetting" period or prior to the maze exposure on day 30. Substantial forgetting was clearly evident as rats made significantly more errors on day 30 than on day 5 , when the asymptote performance was reached (repeated-measures ANOVA, day effect: $F_{(1,26)}$ $=40.607, P<0.001$ ) (Fig. 4). However, memory decay was equal in all experimental groups $\left(F_{(2,26)}=0.960, n s\right)$, there was also no significant interaction $\left(F_{(2,26)}=1.886\right.$, ns) (Fig. 4$)$. Although, on day 30 rats made even more errors as on day $1(6.4 \pm 2.5$ vs. $4.9 \pm 2.9$ $P<0.05)$, more detailed analysis of rat behavior revealed some important differences. First, on day 30 rats actively explored the maze

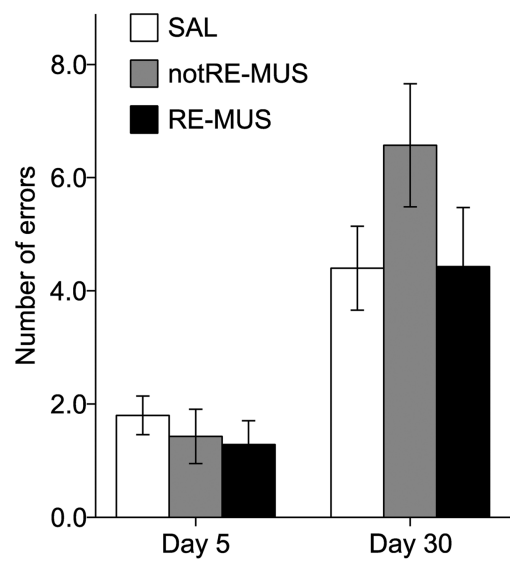

Figure 4. Reversible inactivation of the RE after each of the four task acquisition sessions had no effect on short- or long-term memory retention. The accuracy of task performance during the first trial on day 5 (memory retrieval test $1 \mathrm{~d}$ after the task acquisition) and on day 30 (remote memory retrieval) is shown for three experimental conditions. No drug injection was made during 20-d "forgetting" period or prior memory retrieval tests. Memory expression on days 5 and 30 was equal across three experimental groups. Error bars represent \pm SEM. and most of them reached reward within 3-min cut-off time (SAL: 73.3\%, RE-MUS: $85.7 \%$, notRE-MUS: $42.9 \%$ ). Importantly, the proportion of completed trials on day 30 was much higher than on the very first learning trial on day 1 (6.9\%, all rats combined); the latter indicated that rat behavior on day 30 was clearly target-oriented. Second, the average length of correct path (traversing along the correct trajectory without deviation) was much longer on day 30 $(3.8 \pm 1.5$ maze sections) than on day $1(1.3 \pm 0.3$ maze sections $)$ and, in fact, it was comparable to the performance on day 3 (4.4 \pm 1.9 maze sections). Thus, despite substantial memory decay on day 30 , the rat behavior on the maze was not random and indicated that memory about the task was, at least, partially preserved. Finally, the trial latency on day 30 greatly varied across rats. The latencies distribution was bimodal with peaks around 63 and 162 sec. Using the K-means clustering, we assigned the rats to "slow" and "fast" performers. The "fast" performers showed a somewhat better preserved memory as they made fewer errors $(2.55 \pm 1.57$ vs. $6.39 \pm 2.55$ for "fast" and "slow" performers, respectively; $\left.t_{(26.978)}=5.024, P<0.001\right)$ and had a shorter trajectory $(18.0 \pm 5.9$ vs. $45.3 \pm 11.2$ maze sections, $\left.t_{(27)}=7.425, P<0.001\right)$. However, the proportion of "fast" performers was similar between three groups (SAL: $40.00 \%$, notRE-MUS: $28.57 \%$, and RE-MUS: $42.86 \%$, Kruskal-Wallis test for independent samples, ns).

\section{The RE is critical for spatial memory retrieval}

To assess the effects of RE inactivation on the retrieval of recently acquired spatial memory, we selected rats with injection centers in the RE and with no MUS-induced motor deficits during the retrieval trial $(n=16)$ (Fig. 2). Given that post-learning inactivation of the RE did not affect task acquisition (Fig. 3), we combined rats which received either SAL or MUS injections into the RE during the initial learning phase (days 1-5). After five learning sessions, on days 6 and 7 rats received either MUS or SAL injection 30 min before the maze exposure (Fig. 1B). The RE inactivation dramatically affected the rat behavior on the maze. On the first trial, 9 out of 16 rats $(56.3 \%)$ did not find the reward location within a 3-min cut-off time. The first trial latency was significantly longer compared to SAL injection (149.9 $\pm 45.4 \mathrm{sec}$ vs. $41.9 \pm 38.5 \mathrm{sec}$ after MUS and SAL injection, respectively; $\left.t_{(15)}=-8.4, P<0.001\right)$. Notably, the MUS-injected rats were actively exploring the maze (Fig. 5A). The 
A

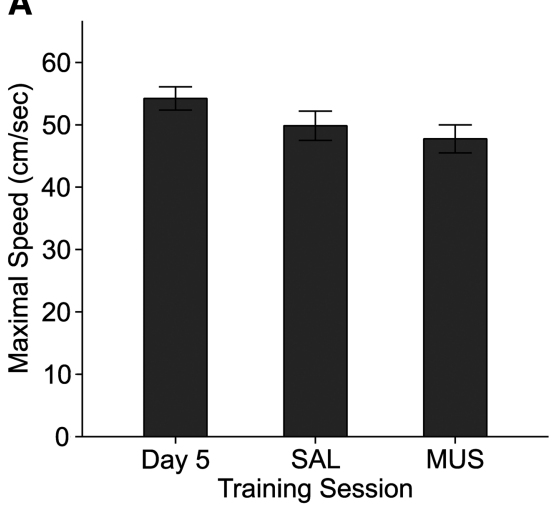

B

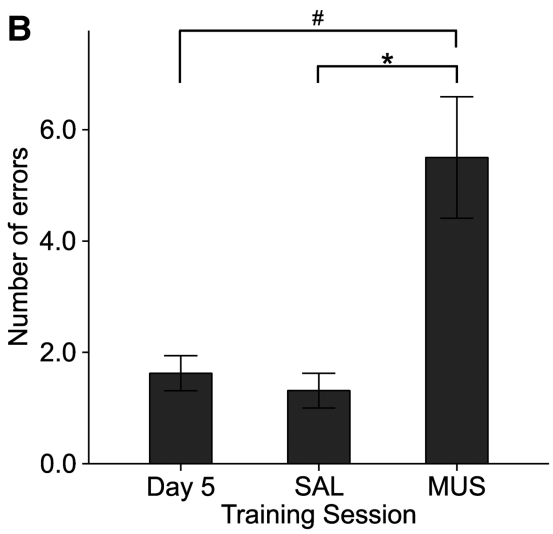

Figure 5. The RE inactivation impairs performance of a spatial task. ( $A$ ) The maximal movement speed on the maze during the drug-free session (day 5, $n=16$ ), after intrabrain injection of SAL $(n=16)$, and after MUS injection in the RE $(n=16)$. (B) The number of errors was significantly higher in the MUS-group. (*) $P<0.01$ and (\#) $P<0.05$ (Bonferroni corrected). Error bars represent \pm SEM.

in both spatial and nonspatial tasks. It is also unlikely that the RE inactivation impaired the rat ability for stimulus-response association as an earlier study showed no effect when the RE-lesioned rats were tested in a visually guided task (Hembrook and Mair 2011).

Our findings may appear to contradict the result of other studies that revealed no effects of the RE inactivation on performance of the Morris water maze (MWM) task (Loureiro et al. 2012; Cholvin et al. 2013). Yet, the discrepancy in the results may actually be explained by the task- and time-specific activation of the memory supporting large-scale network. It is well established that learning of the MWM task depends on the HPC, but does not require the PFC (de Bruin et al. 1994; Sloan et al. 2006). Notably, the recruitment of the PFC is required

trajectory length was twice as long after the RE inactivation (33.0 \pm 17.2 vs. $16.0 \pm 11.1$ maze sections for MUS and SAL, respectively; $\left.t_{(15)}=-4.6, P<0.001\right)$. Remarkably, the MUS-induced performance deficit was preserved during all 10 trials of the maze session (Supplemental Fig. S5). To quantify this observation, we restricted the analysis to rats with unaffected motor activity (Supplemental Fig. S3, Cluster 1). The number of errors was stable across trials and significantly lower on day 5 or after SAL injection than after MUS injection (repeated ANOVA, trials: $F_{(1.419,21.28)}=0.864, \mathrm{~ns}$ ). Thus, the RE inactivation caused a persistent spatial memory deficit as the task performance did not improve with repeated trials.

\section{Discussion}

The findings of the present study suggest that nucleus RE has a selective regulatory effect on the network that support the active phase of learning (information retrieval and encoding), but not involved in the mechanisms of systems consolidation occurring after learning or "off-line." Specifically, we found that the RE inactivation after each of the five learning sessions did not affect the rat's ability to acquire a spatial task, nor did it result in a faster memory decay over a 20-d "forgetting" period. In contrast, the RE inactivation in well-trained rats prior the maze exposure strongly impaired their task performance. Notably, the unaffected motor activity and appetitive motivation were both suggestive of a memory retrieval deficit. Strikingly, there was no improvement in the task performance across subsequent trials of the same session; thus, neither "delayed" memory retrieval (e.g., after first few trials) nor relearning occurred in the RE-inactivated rats. It is possible, that the RE inactivation affected the ability to navigate the maze or to make goal-directed decisions. Indeed, at the start position a rat was required first to localize itself in the environment, likely by using intra- and extramaze cues, then "retrieve" the reward location and navigate along the shortest trajectory based on previously acquired (and stored) information. At each maze crossing, a decisionmaking process (e.g., turn right or left) could, in principle, take place based on the rat's current position and information stored in working memory and/or retrieved from remote memory. However, deficit in navigation or decision-making would also affect spatial learning, which remains largely preserved after the RE lesion or inactivation (Dolleman-van der Weel et al. 2009; Loureiro et al. 2012; Ito et al. 2015). The intriguing possibility of the RE involvement in decision-making or in cognitive flexibility shall be certainly addressed in future studies by testing animals for the retrieval of remote (25-30 d after learning), but not recent (1-5 d) memory in the MWM (Teixeira et al. 2006; Lopez et al. 2012); the latter is consistent with time-dependent reorganization of the circuit supporting memory storage (Frankland and Bontempi 2005). Interestingly, in a modified version of the MWM task, namely, under partial-cue conditions, the PFC is required also for recent memory retrieval (Jo et al. 2007). Furthermore, when rats are tested on a double-H water maze or on a T-maze, both the HPC and the PFC are engaged in the spatial task performance (Cholvin et al. 2013; Layfield et al. 2015). Therefore, the RE inactivation appears ineffective when the HPC-PFC interaction is not required for behavioral execution. Besides, as noted by Hembrook et al. (2012), cognitive demands of the MWM maze task may be insufficient to reveal less pronounced memory deficits like, for example, in cases of lesion of the ventral midline thalamic nuclei. Collectively, our results suggest that the RE may critically contribute to the retrieval of spatial memory and, possibly, to spatial navigation; yet it does not appear to be involved at least in the early phase of "off-line" processing leading to memory stabilization. Our results also support the view that the $\mathrm{RE}$ is important for cognitive functions that depend on the HPC-PFC interaction (Hembrook et al. 2012; Cholvin et al. 2013, 2016; Layfield et al. 2015); memory retrieval, working memory, and spatial navigation belonging to such brain functions (Churchwell et al. 2010; Benchenane et al. 2011; Gordon 2011; Eichenbaum 2017).

The behavioral effects of the RE inactivation reported here support the idea that the RE may coordinate the PFC-HPC interactions. Specifically, gamma-range synchronization between the HPC and PFC is thought to mediate encoding and updating task-related spatial information (Spellman et al. 2015). The PFCHPC synchrony at slower $(4-12 \mathrm{~Hz})$ frequency range presumably facilitates integration and maintenance of information in working memory (Jones and Wilson 2005). The spike-phase coherence and cross-frequency coupling between the HPC and PFC have been attributed to memory encoding (Siapas et al. 2005; Benchenane et al. 2010). Finally, both HPC and PFC function is critical for memory retrieval (Churchwell et al. 2010; Lopez et al. 2012; Cholvin et al. 2016).

The following mechanisms described by now in the literature can account for our results: (1) the excitatory input from the RE may facilitate the HPC-PFC coupling (Dolleman-van der Weel et al. 1997; Di Prisco and Vertes 2006; Hallock et al. 2016; Roy et al. 2017); (2) the neural activity in the CA1 may be adjusted via the HPC output to the RE (Dolleman-van der Weel et al. 1997); (3) 
the RE output may affect spatial coding in the HPC and/or spatial information from the HPC to the PFC may be transferred via the RE (Jankowski et al. 2014; Ito et al. 2015). Besides, the RE, as a part of the circuitry mediating top-down control of dopamine neurons in the ventral tegmental area (Zimmerman and Grace 2016), may influence reward-motivated behaviors. Therefore, if the RE, indeed, gates the bidirectional information flow within the HPC-PFC pathway, it is not surprising that the inactivation of RE impaired the spatial task performance, which is dependent on this functional circuit.

It is also possible that the RE contributes to the systems consolidation, but the RE inactivation in our experiments was not sufficient to cause interference of "off-line" processing. Most studies are consistent in reporting that the effects of MUS reach maximum within 30 min after injection and last for at least $2 \mathrm{~h}$ (Martin 1991; Edeline et al. 2002; Allen et al. 2008). Depending on the injection volume and concentration, it has been shown that the effects of MUS injection can last up to $6 \mathrm{~h}$ (Brandon et al. 2011). In our study, we made an effort to make a rather small injection by using MUS concentration of $0.27 \mu \mathrm{g} / \mu \mathrm{L}$ and volume $0.19 \mu \mathrm{L}$. The acute effects of MUS injection (30-60 min) were rather robust as reflected by impaired task performance on the memory retrieval test or by motor deficit in case of injection outside the RE. The diffusion of fluorophore-conjugated MUS was $\sim 1 \mathrm{~mm}$ radius, which was likely smaller than the diffusion of unlabeled MUS. Taking into account MUS diffusion over time (Edeline et al. 2002), it is unlikely that inactivation of the RE was insufficient; moreover, the adjacent brain regions including the rhomboid nucleus were likely affected by MUS. A higher concentration of MUS or a larger volume would compromise even more the spatial selectivity of the affected brain area. In our experiments, the RE activity was substantially suppressed within at least the first hour after learning when the experience-induced neuronal ensembles replay (Wilson and McNaughton 1994; Peyrache et al. 2009) and other learning-induced changes of HPC and PFC population activity occur (Eschenko et al. 2006, 2008). Consistently, several studies reported effects on memory consolidation due to manipulation of neural activity during the first hour after learning experience (Girardeau et al. 2009; Ego-Stengel and Wilson 2010; Maingret et al. 2016; Novitskaya et al. 2016). It is possible that the duration of the RE inactivation was insufficient to interfere with the mechanisms of systems consolidation or the RE activity may be critical within a delayed post-learning time window.

Finally, any behavioral study of memory unavoidably faces methodological drawbacks, which may complicate the interpretation of results. We used the crossword-like maze, which belongs to a family of multi-unit T-mazes (Tolman 1948) or can be considered as one of configurations of the Hebb-Williams maze (Hebb and Williams 1946); both mazes have been designed and intensively used for studying spatial cognition in rodents. Our experimental design fulfilled the requirements for allocentric navigation (Vorhees and Williams 2014). Successful task performance required spatial orientation at the start position, retrieval of acquired cognitive/spatial map, and use of path integration for updating the animal current position on the maze. The essential role of the HPC in spatial learning and memory is well-established; there is also substantial evidence that the PFC is involved in goal-directed spatial navigation (Porter and Mair 1997; Ragozzino et al. 1998; Dias and Aggleton 2000; Hok et al. 2005; Fujisawa et al. 2008; Churchwell et al. 2010). Moreover, extensive literature exists that learning and performance of various maze tasks depends on the HPC-PFC interaction (Floresco et al. 1997; Jones and Wilson 2005; Benchenane et al. 2010; Hyman et al. 2010; Gordon 2011). The task performance deficit after the RE inactivation observed in our study is consistent with a notion that the RE contribution is critical for the cognitive functions requiring coordinated activa- tion of both the HPC and the PFC (Hembrook et al. 2012; Cholvin et al. 2013; Layfield et al. 2015). However, the involvement of other memory types (e.g., working or procedural) was also-in principle - possible. The working memory, for instance, may have played a role for storing information about visited maze alleys on a given trial. The engagement of procedural memory (e.g., remembering the sequence of turns) cannot be excluded, however it would only be expected at later stages of learning (Packard and McGaugh 1996). The task performance based on procedural memory may be, indeed, advantageous in a stable and highly predictable environment; yet, for example, the action sequence learning does not depend on the RE activity (Hembrook and Mair 2011). Finally, parallel use of different memory systems and navigation strategies could also take place (Iglói et al. 2009). The retrieval of cognitive map may speed up animal orientation at the start position, memory about the maze configuration may facilitate path integration, maintenance in working memory information about visited locations may help for updating the animal current position, and contextual decision-making would help targeted navigation. This scenario is generally consistent with the role of the RE for performance of working memory-dependent spatial tasks (Hembrook and Mair 2011; Hembrook et al. 2012; Hallock et al. 2013, 2016; Layfield et al. 2015). The aforementioned methodological concerns open a possibility that a compromised spatial behavior after the RE inactivation was due to an integrated deficit in the retrieval of spatial memory, in the working memory, in the ability to navigate or make decisions. To further clarify the nature of the behavioral deficit produced by the RE inactivation, testing animals in various situations differing by predominant cognitive demands is crucial.

All in all, our findings further support the hypothesis that the RE is critical for spatial navigation and memory retrieval, yet do not provide evidence for the RE involvement in the mechanisms of systems consolidation, at least during the time window immediately following learning experience.

\section{Materials and Methods}

Male Sprague Dawley rats (Envigo, Huntingdon, UK) weighing 300-350 g at the beginning of experiment were kept in pairs with food and water ad libitum on $12 \mathrm{~h} / 12 \mathrm{~h}$ light-dark cycle. All the experiments were performed during the dark cycle. When rat appetitive behavior was tested, rats were kept on a food-restricted diet to ensure their appetitive motivation at times of behavioral testing. On these days, in addition to the chocolate milk (0.6 $\mathrm{mL}$ ) obtained as reward during maze exposure, each rat received 15-20 g of food pellets and unlimited access to water in their home cage. Rat weight was monitored on a daily basis and kept at $\sim 90 \%$ of ad libitum body weight. All experimental procedures were approved by the local authorities (Regierungspräsidium Tübingen, Germany, Referat 35, Veterinärwesen) in accordance with the regional animal welfare committee pursuant to $\$ 15$ of the German Animal Welfare Act (Kommission nach \$15 des Tierschutzgesetzes), and were in full compliance with the Directive 2010/63/EU of the European Parliament and of the council on the protection of animals used for scientific purposes.

\section{Surgical procedures}

We performed surgeries following standard aseptic procedures. Briefly, before surgery rats were deeply anesthetized with isoflurane (4\% induction, $\sim 1.5 \%$ maintenance) and placed in a stereotaxic frame (David Kopf Instruments). Subsequently, the skull was exposed, a burr hole was drilled to target the RE using the following stereotaxic coordinates: $\mathrm{AP} / \mathrm{ML}=-1.8 /-1.5 \mathrm{~mm}$ (Paxinos and Watson 2005). Furthermore, a stainless-steel guide tube (22-gauge, Plastics One Inc.) was inserted $4.9 \mathrm{~mm}$ deep relative to the surface of the brain and at a medial-lateral angle of $10^{\circ}$ to avoid damaging the sagittal sinus. The guide tube was fixed to the skull using dental 
cement and stainless-steel anchor screws. After the end of the surgery we placed a dummy cannula inside the guide tube to prevent the brain tissue growth. Rats were allowed to have a 1-wk postsurgery recovery before behavioral testing began.

\section{Behavioral apparatus and training procedures}

The crossword maze $(130 \times 130 \mathrm{~cm})$ was custom-built from black polyvinyl chloride. The perpendicular maze alleys $(4 \times 4)$ formed nine identical square sections (Fig. 1A). Maze alleys were $10-\mathrm{cm}$ wide and had $2-\mathrm{cm}$ high rims on both sides. There were two start locations and one reward port connected via tubing with a pump (Izmatec) releasing liquid reward (chocolate milk). To reduce the number of alternative routes on the maze we placed nine vertical barriers (30-cm high and $25-40-\mathrm{cm}$ wide) at specific crossing points, thus restricting the access of the animal to some maze sections. The maze was elevated $80 \mathrm{~cm}$ above the floor and surrounded by black curtains; two posters served as extramaze visual cues. All behavioral procedures were performed under dim light.

For habituation, the maze was converted to a single linear alley. A rat was released from one end of the alley, had to reach the reward port at the other end and obtain reward by nose poking. This procedure was repeated until the rat behavior became consistently reward oriented. The start and reward locations were different from the ones used for the main task; extramaze visual cues were removed. Rats were also habituated to the intrabrain microinjection procedure by handling. After three habituation sessions, rats were trained on a spatial task for five consecutive days. Two start locations were used in pseudo-random order to minimize the procedural component of learning; the two shortest (correct) trajectories leading to reward partially overlapped (Fig. 1A). A rat was released from the start location and allowed 3 min to reach the reward port. After reward consumption or after the trial cut-off time elapsed, the rat was removed from the maze and left in a waiting box for 3-5 min. During each intertrial interval the maze alleys were wiped to minimize local olfactory cues. Each training session consisted of 10 trials. Immediately after the learning session rats received either intrabrain injection of phosphate-buffered saline (SAL-group) or muscimol (MUS-group) and returned to their home cages. On days 6 and 7, rats received either SAL or MUS injection 30 min before the maze exposure (Fig. 1B). On days 8 and 9 , rats were trained on the same task (without any drug injections) to further stabilize the acquired spatial memory and then kept in their home cages for $20 \mathrm{~d}$ without any behavioral testing; during this "forgetting" period rats had food and water ad libitum, except $24 \mathrm{~h}$ before the remote memory retrieval test (Fig. 1B).

\section{Intrabrain microinjection}

The MUS powder (Sigma-Aldrich) was diluted in SAL at a final concentration of $0.27 \mu \mathrm{g} / \mu \mathrm{L}$. The injections were performed using Hamilton syringe loaded into a microinfusion pump (UMP3, WPI). For the drug injection procedure, a rat was gently restrained by hand, the dummy cannula was removed and the injection cannula was inserted inside the guide tube. The tip of the injection cannula extended $2 \mathrm{~mm}$ beyond the tip of the guide tube. We used polyethylene tubing to connect the injection cannula to the Hamilton syringe. MUS or SAL $(0.19 \mu \mathrm{L})$ was infused over 60 sec. The injection cannula was left in place for additional 2 min before it was slowly retracted. The dummy cannula was then inserted back to the guide tube.

\section{Data analysis}

The rat behavior on the maze was video recorded by an infrared camera. The trial time was measured manually using a stopwatch. The rat trajectories on the maze were reconstructed manually from video and the movement speed was extracted and further analyzed using custom made program in MATLAB (MathWorks). Any deviation from the "correct" (shortest) trajectory leading to reward was considered as an error. Specifically, if a rat entered a maze alley, which did not belong to the "correct" path, with all four limbs, it was scored as an error. Further animal passage along other maze al- leys outside of the "correct" path after deviating was not scored as additional errors unless the rat returned to the correct path (see examples on Supplemental Fig. S1A). Moving along the correct trajectory, but in opposite direction (away from reward port) was also considered as an error (see examples on Supplemental Fig. S1B). The trajectory length, the time to reach reward, and the number of errors were extracted for each trial. To assess the "content" of spatial memory from the rat trajectory, maze alleys were arbitrary divided into 24 equal-length sections separated by maze crossings; each maze section was assigned a unique number and the movement sequences analyzed. Thus, the shortest (correct) trajectory from the Start 1 (S1) consisted of nine maze sections (Fig. 1A). For each trial, we extracted the mean length of the rat trajectory (number of maze sections) overlapping with the correct path before deviation from the correct path.

Behavioral variables during five learning sessions were submitted to the repeated-measures ANOVA; post hoc comparisons were made when appropriate using Bonferroni correction. Data were tested for equality of error variance and Greenhouse-Geisser correction was applied whenever the assumption of sphericity was violated. The one-way ANOVA or Student $t$-test was used for between-group comparisons. The statistical significance $\alpha$-value was set at $P<0.05$ level. The IBM SPSS Statistics (v.22) software package was used for statistical analysis.

\section{Perfusion and histology}

At the end of behavioral experiments, we injected each rat with fluorophore-conjugated MUS (Thermo Fisher Scientific). This helped to localize the site of injection and evaluate the extent of drug diffusion. After $\sim 30$ min post-injection, the rat was euthanized with a lethal dose of sodium pentobarbital $(100 \mathrm{mg} / \mathrm{kg}$ i.p.; Narcoren, Merial $\mathrm{GmbH}$ ) and perfused transcardially with $0.9 \%$ saline followed by $4 \%$ paraformaldehyde in $0.1 \mathrm{M}$ phosphate buffer $(\mathrm{PB}, \mathrm{pH}$ 7.4). The brain was removed and stored in the same fixative. Before sectioning, the whole brain was placed in 30\% sucrose solution at $4^{\circ} \mathrm{C}$ until they sank. Serial $50-\mu \mathrm{m}$-thick coronal sections were cut on a horizontal freezing microtome (Thermo Fisher Scientific). Every second section was Nissl-stained; adjacent sections were stored for examination under the fluorescent microscope (AxioVision, Carl Zeiss). Position of the injection cannula tip was assessed visually and digitized.

\section{Acknowledgments}

We thank Johannes Boldt, Jannik Romanowski and Oliver Holder for building the crossword maze, Leyla Maharramli for help with behavioral experiment, Katalin Kalya for help with histology, Eduard Krampe, Axel Oeltermann, and Joachim Werner for technical support, Daniel Zaldivar and Mingyu Yang for comments on earlier version of the manuscript.

\section{References}

Ali M, Cholvin T, Muller MA, Cosquer B, Kelche C, Cassel JC, Pereira de Vasconcelos A. 2017. Environmental enrichment enhances systemslevel consolidation of a spatial memory after lesions of the ventral midline thalamus. Neurobiol Learn Mem 141: 108-123.

Allen TA, Narayanan NS, Kholodar-Smith DB, Zhao Y, Laubach M, Brown TH. 2008. Imaging the spread of reversible brain inactivations using fluorescent muscimol. J Neurosci Methods 171: 30-38.

Benchenane K, Peyrache A, Khamassi M, Tierney PL, Gioanni Y, Battaglia FP, Wiener SI. 2010. Coherent $\theta$ oscillations and reorganization of spike timing in the hippocampal-prefrontal network upon learning. Neuron 66: 921-936.

Benchenane K, Tiesinga PH, Battaglia FP. 2011. Oscillations in the prefrontal cortex: a gateway to memory and attention. Curr Opin Neurobiol 21: $475-485$.

Brandon MP, Bogaard AR, Libby CP, Connerney MA, Gupta K, Hasselmo ME. 2011. Reduction of $\theta$ rhythm dissociates grid cell spatial periodicity from directional tuning. Science 332: 595-599.

Buzsáki G. 1996. The hippocampo-neocortical dialogue. Cereb Cortex 6: 81-92.

Buzsáki G, Moser EI. 2013. Memory, navigation and $\theta$ rhythm in the hippocampal-entorhinal system. Nat Neurosci 16: 130-138. 
Cholvin T, Loureiro M, Cassel R, Cosquer B, Geiger K, De Sa Nogueira D, Raingard H, Robelin L, Kelche C, Pereira de Vasconcelos A, et al. 2013. The ventral midline thalamus contributes to strategy shifting in a memory task requiring both prefrontal cortical and hippocampal functions. J Neurosci 33: 8772-8783.

Cholvin T, Loureiro M, Cassel R, Cosquer B, Herbeaux K, de Vasconcelos AP, Cassel JC. 2016. Dorsal hippocampus and medial prefrontal cortex each contribute to the retrieval of a recent spatial memory in rats. Brain Struct Funct 221: 91-102.

Churchwell JC, Morris AM, Musso ND, Kesner RP. 2010. Prefrontal and hippocampal contributions to encoding and retrieval of spatial memory. Neurobiol Learn Mem 93: 415-421.

Colgin LL. 2011. Oscillations and hippocampal-prefrontal synchrony. Curr Opin Neurobiol 21: 467-474.

Davoodi FG, Motamedi F, Naghdi N, Akbari E. 2009. Effect of reversible inactivation of the reuniens nucleus on spatial learning and memory in rats using Morris water maze task. Behav Brain Res 198: 130-135.

Davoodi FG, Motamedi F, Akbari E, Ghanbarian E, Jila B. 2011. Effect of reversible inactivation of reuniens nucleus on memory processing in passive avoidance task. Behav Brain Res 221: 1-6.

de Bruin JPC, Sànchez-Santed F, Heinsbroek RPW, Donker A, Postmes P. 1994. A behavioural analysis of rats with damage to the medial prefrontal cortex using the morris water maze: evidence for behavioural flexibility, but not for impaired spatial navigation. Brain Res 652: 323-333.

Dias R, Aggleton JP. 2000. Effects of selective excitotoxic prefrontal lesions on acquisition of nonmatching- and matching-to-place in the T-maze in the rat: differential involvement of the prelimbic-infralimbic and anterior cingulate cortices in providing behavioural flexibility. Eur Neurosci 12: 4457-4466.

Di Prisco GV, Vertes RP. 2006. Excitatory actions of the ventral midline thalamus (rhomboid/reuniens) on the medial prefrontal cortex in the rat. Synapse 60: $45-55$.

Dolleman-van der Weel MJ, Lopes da Silva FH, Witter MP. 1997. Nucleus reuniens thalami modulates activity in hippocampal field CA1 through excitatory and inhibitory mechanisms. J Neurosci 17: 5640-5650.

Dolleman-van der Weel M, Morris RM, Witter M. 2009. Neurotoxic lesions of the thalamic reuniens or mediodorsal nucleus in rats affect nonmnemonic aspects of watermaze learning. Brain Struct Funct 213: 329-342.

Edeline J-M, Hars B, Hennevin E, Cotillon N. 2002. Muscimol diffusion after intracerebral microinjections: a reevaluation based on electrophysiological and autoradiographic quantifications. Neurobiol Learn Mem 78: 100-124.

Ego-Stengel V, Wilson MA. 2010. Disruption of ripple-associated hippocampal activity during rest impairs spatial learning in the rat. Hippocampus 20: 1-10.

Eichenbaum H. 2017. Prefrontal-hippocampal interactions in episodic memory. Nat Rev Neurosci 18: 547-558.

Eschenko O, Moelle M, Born J, Sara SJ. 2006. Elevated sleep spindle density after learning or after retrieval in rats. J Neurosci 26: 12914-12920.

Eschenko O, Ramadan W, Mölle M, Born J, Sara SJ. 2008. Sustained increase in hippocampal sharp-wave ripple activity during slow-wave sleep after learning. Learn Mem 15: 222-228.

Floresco SB, Seamans JK, Phillips AG. 1997. Selective roles for hippocampal, prefrontal cortical, and ventral striatal circuits in radial-arm maze tasks with or without a delay. J Neurosci 17: 1880-1890.

Frankland PW, Bontempi B. 2005. The organization of recent and remote memories. Nat Rev 6: 119-130.

Fujisawa S, Amarasingham A, Harrison MT, Buzsáki G. 2008. Behaviordependent short-term assembly dynamics in the medial prefrontal cortex. Nat Neurosci 11: 823-833.

Girardeau G, Benchenane K, Wiener SI, Buzsáki G, Zugaro MB. 2009. Selective suppression of hippocampal ripples impairs spatial memory. Nat Neurosci 12: 1222-1223.

Gordon JA. 2011. Oscillations and hippocampal-prefrontal synchrony. Curr Opin Neurobiol 21: 486-491.

Griffin AL. 2015. Role of the thalamic nucleus reuniens in mediating interactions between the hippocampus and medial prefrontal cortex during spatial working memory. Front Syst Neurosci 9: 29.

Hallock HL, Wang A, Shaw CL, Griffin AL. 2013. Transient inactivation of the thalamic nucleus reuniens and rhomboid nucleus produces deficits of a working-memory-dependent tactile-visual conditional discrimination task. Behav Neurosci 127: 860-866.

Hallock HL, Wang A, Griffin AL. 2016. Ventral midline thalamus is critical for hippocampal-prefrontal synchrony and spatial working memory. $J$ Neurosci 36: 8372-8389.

Hebb DO, Williams K. 1946. A method of rating animal intelligence. J Gen Psychol 34: 59-65.

Hembrook JR, Mair RG. 2011. Lesions of reuniens and rhomboid thalamic nuclei impair radial maze win-shift performance. Hippocampus 21: 815-826.
Hembrook JR, Onos KD, Mair RG. 2012. Inactivation of ventral midline thalamus produces selective spatial delayed conditional discrimination impairment in the rat. Hippocampus 22: 853-860.

Hok V, Save E, Lenck-Santini PP, Poucet B. 2005. Coding for spatial goals in the prelimbic/infralimbic area of the rat frontal cortex. Proc Natl Acad Sci 102: $4602-4607$.

Hoover WB, Vertes RP. 2007. Anatomical analysis of afferent projections to the medial prefrontal cortex in the rat. Brain Struct Funct 212: 149-179.

Hoover W, Vertes R. 2012. Collateral projections from nucleus reuniens of thalamus to hippocampus and medial prefrontal cortex in the rat: a single and double retrograde fluorescent labeling study. Brain Struct Funct 217: $191-209$

Hyman JM, Zilli EA, Paley AM, Hasselmo ME. 2005. Medial prefrontal cortex cells show dynamic modulation with the hippocampal $\theta$ rhythm dependent on behavior. Hippocampus 15: 739-749.

Hyman J, Zilli E, Paley A, Hasselmo M. 2010. Working memory performance correlates with prefrontal-hippocampal theta interactions but not with prefrontal neuron firing rates. Front Integr NeurosciFrontiers in Integrative Neuroscience 4: 2.

Iglói K, Zaoui M, Berthoz A, Rondi-Reig L. 2009. Sequential egocentric strategy is acquired as early as allocentric strategy: parallel acquisition of these two navigation strategies. Hippocampus 19: 1199-1211.

Ito HT, Zhang S-J, Witter MP, Moser EI, Moser M-B. 2015. A prefrontalthalamo-hippocampal circuit for goal-directed spatial navigation. Nature 522: $50-55$.

Jankowski MM, Islam MN, Wright NF, Vann SD, Erichsen JT, Aggleton JP, O'Mara SM. 2014. Nucleus reuniens of the thalamus contains head direction cells. Elife 3: e03075.

Jo YS, Park EH, Kim IH, Park SK, Kim H, Kim HT, Choi J-S. 2007. The medial prefrontal cortex is involved in spatial memory retrieval under partialcue conditions. J Neurosci 27: 13567-13578.

Jones MW, Wilson MA. 2005. Theta rhythms coordinate hippocampalprefrontal interactions in a spatial memory task. PLoS BiolPLoS biology 3: e402.

Layfield DM, Patel M, Hallock H, Griffin AL. 2015. Inactivation of the nucleus reuniens/rhomboid causes a delay-dependent impairment of spatial working memory. Neurobiol Learn Mem 125: 163-167.

Lopez J, Herbeaux K, Cosquer B, Engeln M, Muller C, Lazarus C, Kelche C, Bontempi B, Cassel JC, de Vasconcelos AP. 2012. Context-dependent modulation of hippocampal and cortical recruitment during remote spatial memory retrieval. Hippocampus 22: 827-841.

Loureiro M, Cholvin T, Lopez J, Merienne N, Latreche A, Cosquer B, Geiger K, Kelche C, Cassel J-C, Pereira de Vasconcelos A. 2012. The ventral midline thalamus (reuniens and rhomboid nuclei) contributes to the persistence of spatial memory in rats. J Neurosci 32: 9947-9959.

Maingret N, Girardeau G, Todorova R, Goutierre M, Zugaro M. 2016. Hippocampo-cortical coupling mediates memory consolidation during sleep. Nat Neurosci 19: 959-964.

Martin JH. 1991. Autoradiographic estimation of the extent of reversible inactivation produced by microinjection of lidocaine and muscimol in the rat. Neurosci Lett 127: 160-164.

McKenna JT, Vertes RP. 2004. Afferent projections to nucleus reuniens of the thalamus. J Comp Neurol 480: 115-142.

McNaughton BL, Battaglia FP, Jensen O, Moser EI, Moser MB. 2006. Path integration and the neural basis of the 'cognitive map'. Nat Rev Neurosci 7: 663-678.

Mizumori SJY, Cooper BG, Leutgeb S, Pratt WE. 2000. A neural systems analysis of adaptive navigation. Mol Neurobiol 21: 57-82.

Moelle M, Eschenko O, Gais S, Sara SJ, Born J. 2009. The influence of learning on sleep slow oscillations and associated spindles and ripples in humans and rats. Eur J Neurosci 29: 1071-1081.

Novitskaya Y, Sara SJ, Logothetis NK, Eschenko O. 2016. Ripple-triggered stimulation of the locus coeruleus during post-learning sleep disrupts ripple/spindle coupling and impairs memory consolidation. Learn Mem 23: $238-248$.

Packard MG, McGaugh JL. 1996. Inactivation of hippocampus or caudate nucleus with lidocaine differentially affects expression of place and response learning. Neurobiol Learn Mem 65: 65-72.

Paxinos G, Watson C. 2005. The rat brain in stereotaxic coordinates. Elsevier Academic Press, Amsterdam; Boston.

Peyrache A, Khamassi M, Benchenane K, Wiener SI, Battaglia FP. 2009. Replay of rule-learning related neural patterns in the prefrontal cortex during sleep. Nat Neurosci 12: 919-926.

Porter MC, Mair RG. 1997. The effects of frontal cortical lesions on remembering depend on the procedural demands of tasks performed in the radial arm maze. Behav Brain Res 87: 115-125.

Ragozzino ME, Adams S, Kesner RP. 1998. Differential involvement of the dorsal anterior cingulate and prelimbic-infralimbic areas of the rodent prefrontal cortex in spatial working memory. Behav Neurosci 112: 293-303.

Rajasethupathy P, Sankaran S, Marshel JH, Kim CK, Ferenczi E, Lee SY, Berndt A, Ramakrishnan C, Jaffe A, Lo M, et al. 2015. Projections from 
neocortex mediate top-down control of memory retrieval. Nature 526: 653-659.

Roy A, Svensson FP, Mazeh A, Kocsis B. 2017. Prefrontal-hippocampal coupling by $\theta$ rhythm and by $2-5 \mathrm{~Hz}$ oscillation in the $\delta$ band: the role of the nucleus reuniens of the thalamus. Brain Struct Funct 222: 2819-2830.

Siapas AG, Wilson MA. 1998. Coordinated interactions between hippocampal ripples and cortical spindles during slow-wave sleep. Neuron 21: 1123-1128.

Siapas AG, Lubenov EV, Wilson MA. 2005. Prefrontal phase locking to hippocampal $\theta$ oscillations. Neuron 46: 141-151.

Sloan HL, Good M, Dunnett SB. 2006. Double dissociation between hippocampal and prefrontal lesions on an operant delayed matching task and a water maze reference memory task. Behav Brain Res 171: 116-126.

Spellman T, Rigotti M, Ahmari SE, Fusi S, Gogos JA, Gordon JA. 2015. Hippocampal-prefrontal input supports spatial encoding in working memory. Nature 522: 309-314.

Teixeira CM, Pomedli SR, Maei HR, Kee N, Frankland PW. 2006. Involvement of the anterior cingulate cortex in the expression of remote spatial memory. J Neurosci 26: 7555-7564.

Thierry AM, Gioanni Y, Degenetais E, Glowinski J. 2000. Hippocampoprefrontal cortex pathway: anatomical and electrophysiological characteristics. Hippocampus 10: 411-419.

Tolman EC. 1948. Cognitive maps in rats and men. Psychol Rev 55: 189-208.

Varela C, Kumar S, Yang JY, Wilson MA. 2014. Anatomical substrates for direct interactions between hippocampus, medial prefrontal cortex, and the thalamic nucleus reuniens. Brain Struct Funct 219: 911-929.
Vertes RP. 2006. Interactions among the medial prefrontal cortex, hippocampus and midline thalamus in emotional and cognitive processing in the rat. Neuroscience 142: 1-20.

Vertes RP, Hoover WB, Do Valle AC, Sherman A, Rodriguez JJ. 2006. Efferent projections of reuniens and rhomboid nuclei of the thalamus in the rat. $J$ Comp Neurol 499: 768-796.

Vertes RP, Hoover WB, Szigeti-Buck K, Leranth C. 2007. Nucleus reuniens of the midline thalamus: link between the medial prefrontal cortex and the hippocampus. Brain Res Bull 71: 601-609.

Vorhees CV, Williams MT. 2014. Assessing spatial learning and memory in rodents. ILAR J 55: 310-332.

Wierzynski CM, Lubenov EV, Gu M, Siapas AG. 2009. State-dependent spike-timing relationships between hippocampal and prefrontal circuits during sleep. Neuron 61: 587-596.

Wilson MA, McNaughton BL. 1994. Reactivation of hippocampal ensemble memories during sleep. Science 265: 676-679.

$\mathrm{Xu}$ W, Sudhof TC. 2013. A neural circuit for memory specificity and generalization. Science 339: 1290-1295.

Zimmerman EC, Grace AA. 2016. The nucleus reuniens of the midline thalamus gates prefrontal-hippocampal modulation of ventral tegmental area dopamine neuron activity. J Neurosci 36: $8977-8984$.

Received November 18, 2017; accepted in revised form December 19, 2017. 


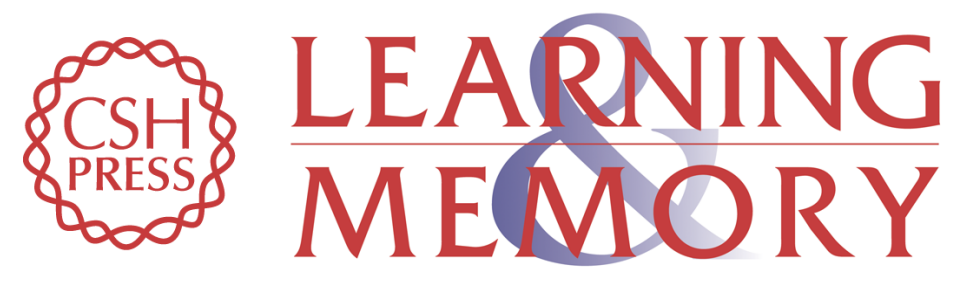

\section{The activity of thalamic nucleus reuniens is critical for memory retrieval, but not essential for the early phase of "off-line" consolidation}

Hao Mei, Nikos K. Logothetis and Oxana Eschenko

Learn. Mem. 2018, 25:

Access the most recent version at doi:10.1101//m.047134.117

\section{Supplemental http://learnmem.cshlp.org/content/suppl/2018/02/09/25.3.129.DC1 \\ Material}

References This article cites 76 articles, 15 of which can be accessed free at: http://learnmem.cshlp.org/content/25/3/129.full.html\#ref-list-1

Creative This article is distributed exclusively by Cold Spring Harbor Laboratory Press for the Commons first 12 months after the full-issue publication date (see License http://learnmem.cshlp.org/site/misc/terms.xhtml). After 12 months, it is available under a Creative Commons License (Attribution-NonCommercial 4.0 International), as described at http://creativecommons.org/licenses/by-nc/4.0/.

Email Alerting Receive free email alerts when new articles cite this article - sign up in the box at the Service top right corner of the article or click here. 